\title{
Preparation and characterization of bismuth germanium oxide (BGO) polymer composites
}

\author{
Ljiljana M. Brajovic a, *, Dusica B. Stojanovic ${ }^{\text {b }}$, Pedja Mihailovic ${ }^{c}$, Smilja B. Markovic ${ }^{\text {d }}$, \\ Maja Romcevic ${ }^{\mathrm{f}}$, Miodrag Mitric ${ }^{\mathrm{e}}$, Vladimir Lazovic ${ }^{\mathrm{f}}$, Dragan Dramlic ${ }^{\mathrm{f}}$, \\ Slobodan Petricevic ${ }^{\mathrm{c}}$, Nebojsa Romcevic ${ }^{\mathrm{f}}$ \\ ${ }^{a}$ University of Belgrade, Civil Engineering Faculty, Bulevar Kralja Aleksandra 73, 11000 Belgrade, Serbia \\ ${ }^{\mathrm{b}}$ University of Belgrade, Faculty of Technology and Metallurgy, Karnegijeva 4, 11120 Belgrade, Serbia \\ c University of Belgrade, Faculty of Electrical Engineering, Bulevar Kralja Aleksandra 73, 11000 Belgrade, Serbia \\ ${ }^{\mathrm{d}}$ University of Belgrade, Institute of Technical Sciences of SASA, Knez Mihailova 35/IV, 11000 Belgrade, Serbia \\ e University of Belgrade, Institute of Nuclear Sciences Vinča, P.O. Box 522, 11001 Belgrade, Serbia \\ ${ }^{\mathrm{f}}$ University of Belgrade, Institute of Physics, P.O. Box 68, Pregrevica 118, Zemun, 11080 Belgrade, Serbia
}

\section{A R T I C L E I N F O}

\section{Article history:}

Received 31 May 2016

Received in revised form

24 September 2016

Accepted 16 October 2016

Available online 17 October 2016

\section{Keywords:}

Composite materials

Polymer

Elastic scattering

Optical properties

Optical spectroscopy

\begin{abstract}
A B S T R A C T
Bismuth germanium oxide $\mathrm{Bi}_{12} \mathrm{GeO}_{20}$ (BGO) has very interesting electrical, optical and magnetic properties. In order to make devices based on this material more flexible, its powder was used for preparing polymer composites. This study reports investigation of the effects of using different solvents and polymers in preparation of BGO composite on the microstructural and optical behaviour of the resulting material. Preparation of such composites by a solution casting method is described. Poly (methyl methacrylate) (PMMA) and polystyrene (PS)) were used as matrix materials and acetone and chloroform as solvents. Their microstructure and the quality of BGO dispersion and deaggregation in polymer matrix were analyzed by SEM, Raman, XRD and optical spectroscopy. The influence of particle size distribution, their shapes, and concentration on the optical transmission is calculated based on Mie scattering theory and discussed, too.
\end{abstract}

(C) 2016 Elsevier B.V. All rights reserved.

\section{Introduction}

As a member of sillenite single crystals, Bismuth germanium oxide, $\mathrm{Bi}_{12} \mathrm{GeO}_{20}$ (BGO) has only one non-bismuth metal atom in a formula unit of 33 atoms. Its structure with only one Ge atom for every $12 \mathrm{Bi}$ atoms and only four of 20 oxygen atoms involved in $\mathrm{GeO}_{4}$ tetrahedron is foundation for many interesting properties such as photoconductivity, magneto-optical effect, electro-optical effect, piezoelectricity, electrogyratory effect and photorefractivity [1-3]. These properties are applicative in the fields of optical sensors, optical memories, holography, etc. [4-8]. Composites with polymer matrix are materials of great interest because their properties can be adjusted by: controlling the content, morphology and composition of the particle reinforcement, different processing techniques and modification of the polymer matrix [9-11].

Using BGO powder as reinforcement for creating composite

\footnotetext{
* Corresponding author. Tel.: + 381113218585.

E-mail address: brajovic@grf.bg.ac.rs (L.M. Brajovic).
}

materials would potentially broaden and technologically improve its applications.

BGO large refraction index compared to the matrix polymers leads to high scattering on powder particles and radiation loss. Although high loss can be acceptable for sensing applications it is of interest to find a suitable technological procedure to make samples homogenous in particle size distribution and more transparent.

In this paper preparation and characterization of composites with poly (methyl methacrylate) (PMMA) or polystyrene (PS) matrix and milled BGO powder as reinforcement are described.

The solution casting method is chosen since it gives the better transparency of the samples compared with melt compounding methods [12]. For PMMA based samples two solvents were used acetone and chloroform, and for preparing PS sample chloroform is used as solvent. Particle size distribution of powder itself and of prepared composites based on their SEM images is used to compare homogeneity of samples, as well as, the size and shapes of their particles and aggregates. X-ray diffraction (XRD) and Raman spectra analysis of the samples were done to authenticate 
powdered single crystal BGO in the composite samples and to investigate how different preparing procedure influence the obtained spectra. Besides characterization of the samples the optical spectroscopy was used for comparing the measured and calculated transmission of samples based on Mie scattering theory.

To the best of our knowledge, the selected $\mathrm{Bi}_{12} \mathrm{GeO}_{20}$-PMMA and $\mathrm{Bi}_{12} \mathrm{GeO}_{20}$-PS composite systems has not been previously reported in the literature.

\section{Experimental}

\subsection{Materials}

$\mathrm{Bi}_{12} \mathrm{GeO}_{20}$ single crystals were grown by the Czochralski technique using a MSR 2 crystal puller controlled by a Eurotherm. The charge for preparing this yellow crystal was a stoichiometric mixture of $\mathrm{Bi}_{2} \mathrm{O}_{3}$ (99.999 wt\%) and $\mathrm{GeO}_{2}$ (99.9999 wt\%). Details of the preparation are presented in Ref. [13]. Powdering of synthesized single crystals was done by milling in planetary high energy ball mill (Fritsch Pulverisettes).

The polymer components of the composites were: a) commercially available heat resistant injection grade PMMA pellets, Acryrex_CM-205 (Mw = 90400, Chi Mei Corporation, Taiwan).with no detected solute according to RoHS Directive, b) commercially available Polystyrene (PS) pellets, Empera ${ }^{\mathbb{B}} 251 \mathrm{~N}$, Ineos Nova, c) acetone purchased at Beta Hemm and d) chloroform purchased at Fisher Scientific from UK.

\subsection{Preparation of composite samples}

The composite samples were prepared with a solution casting method. Three samples with different polymer or solvent are prepared, but all with the same BGO mass fraction of $1.0 \mathrm{wt} \%$.

The first sample (in following text denoted as no.1) was obtained by dissolving PMMA in acetone for $24 \mathrm{~h}$, and then by adding BGO powder to the solution with continuous magnetic stirring. The mixture was poured in the Petri dish through a $5 \mu \mathrm{m}$ mesh sieve to prevent bubbling and left inside an oven at constant temperature of $50{ }^{\circ} \mathrm{C}$ for another $24 \mathrm{~h}$, and then in a vacuum drying oven for $8 \mathrm{~h}$ at $50{ }^{\circ} \mathrm{C}$.

The second sample (no.2) was prepared by dissolving PMMA in chloroform and then adding BGO powder to the solution with continuous bath ultrasound (15 min) and magnetic stirring for $24 \mathrm{~h}$. The mixture was then poured into $50 \mathrm{~mm}$ diameter Petri dishes, left inside an oven at constant temperature of $50{ }^{\circ} \mathrm{C}$ for another $24 \mathrm{~h}$, and then in a vacuum drying oven for $8 \mathrm{~h}$ at $50^{\circ} \mathrm{C}$.

The sample no. 3 was obtained by dissolving Polystyrene (PS) in chloroform and then adding BGO powder to the solution with continuous bath ultrasound ( $15 \mathrm{~min}$ ) and magnetic stirring for $24 \mathrm{~h}$. The mixture was then poured into $50 \mathrm{~mm}$ diameter Petri dishes, and the further procedures were the same as for sample no.2. The thickness of all prepared samples was $0.6 \mathrm{~mm}$.

\subsection{Characterization methods}

The particle size distribution (PSD) of BGO powder was determined by a laser light-scattering particle size analyzer (PSA). The used instrument was Mastersizer 2000 (Malvern Instruments Ltd., UK) particle size analyzer based on laser diffraction, covering the particle size range of $0.02-2000 \mu \mathrm{m}$. For the PSA measurements, the powder was dispersed in distilled water, in ultrasonic bath (low-intensity ultrasound, at a frequency of $40 \mathrm{kHz}$ and power of $50 \mathrm{~W}$ ), for $20 \mathrm{~min}$.

SEM imaging of BGO polymer composites was performed using field emission scanning electron microscope FESEM (TESCAN MIRA
3 ) in order to investigate differences in dispersion and deaggregation of particles in composite samples. The size distribution of particles in the composites was obtained through manual measurements and analysis of SEM images using program Image Pro Plus 6.0 (Media Cybernetics).

$\mathrm{X}$-ray diffraction (XRD) analyses of powdered single BGO crystals and composite samples were performed on a Philips 1050 X-ray powder diffractometer using a $\mathrm{Ni}$-filtered $\mathrm{CuK}_{\alpha}$ radiation and Bragg-Brentano focusing geometry. The patterns were taken in the $10^{\circ}<2 \theta<100^{\circ}$ range with the scanning step of $0.05^{\circ}$ and exposure time of 5 s per step.

The Raman spectra of polymer composites were obtained by the micro-Raman and were analyzed using Jobin Yvon T64000 spectrometer, equipped with nitrogen cooled charge-coupled device detector. The measurements were performed at $20 \mathrm{~mW}$ during $200 \mathrm{~s}$ at room temperature. The spectral range of Raman was from 50 to $900 \mathrm{~cm}^{-1}$, in back scattering geometry.

Optical transmission spectra of the single crystal, composite samples and pure polymer films as a control were measured in VIS and IR ranges using DU 720 General purpose UV-VIS spectrometer (300-1100 nm).

\section{Results and discussion}

\subsection{Powder particle size distribution}

The particle size distribution, based on number, of the analyzed BGO powder is presented in Fig. 1. The particle size distribution was relatively narrow (span $=2.243$ ) where $10 \%$ of particles, $d(0.1)$, have diameter smaller than $0.125 \mu \mathrm{m}, 50 \%$ of particles possess diameter of $d(0.5)=0.240 \mu \mathrm{m}$, while $90 \%$ of particles, $d(0.9)$, are smaller than $0.670 \mu \mathrm{m}$.

\subsection{SEM analysis and obtaining the PSD of composite samples}

Three SEM of sample no.1 with different magnifications $(6.17 \mathrm{k}$, $11.3 \mathrm{k}$ and $40.0 \mathrm{k}$ ) are presented in Fig. 2 . The first one shows the broad area of composite and distribution of various powder particle sizes in composite. The second micrograph shows the marked detail of the first one in order to establish shapes and sizes of various kinds of powder particles and aggregates that are formed. The third one shows structure of aggregates for micrograph detail marked in the second one. It is obvious that the aggregates have mostly round shapes and their size is up to $10 \mu \mathrm{m}$.

Similar three micrographs of sample no. 2 are presented in Fig. 3 whose magnifications were $4.08 \mathrm{k}, 26.1 \mathrm{k}$ and $83.9 \mathrm{k}$ respectively. This sample has more homogeneous structure compared to sample no.1, the particle and aggregate sizes are up to $4 \mu \mathrm{m}$ and their shapes are mostly round.

In Fig. 4, three micrographs of sample no.3 are presented with magnifications of $3.77,8.64 \mathrm{k}$ and $29.1 \mathrm{k}$. The particles and aggregates are pretty uniformly distributed, but their shapes are quite different compared to other two samples. Shapes of aggregates and particles are not spherical in the majority, but more ellipsoidal, sometimes even pyramidal and their size is up to $9 \mu \mathrm{m}$.

Additional SEM micrographs of all sample types are presented in the Supplement data.

In order to compare samples, PSD analysis is obtained. Since the shapes of particles are different as well as their visibility, the analysis was done manually using Image ProPlus 6.0. The measured values denoted as $d$ were the longest dimensions of the particles.

The histograms presenting size count probabilities for all three samples for the sizes up to $8 \mu \mathrm{m}$ are presented in Fig. 5 .

The number of analyzed particles $N$, minimum and maximum observed size, mean size value, standard deviation, as well as 
$\mathrm{d}(0.5)$ and $\mathrm{d}(0.9)$ values are shown in Table 1.

Comparing the powder PSD from paragraph 3.1 and PSDs of prepared samples, it is obvious, that although all preparing procedures have some kind of particles dispersion in the polymer solution, during evaporation of the solvent and forming the composite, the various aggregates of particles were formed. The values of $d(0.5)$ and $d(0.9)$ have higher values for the prepared samples no. 1 and no.3 than in powder PSD which illustrates those statement. The best homogeneity and the smallest aggregates have sample no.2.

\subsection{XRD characterization of pure crystal and composite samples}

XRD characterization was performed to authenticate powdered single crystal BGO in the composite samples. The presented graphs in Fig. 6 show XRD patterns of powdered single BGO crystals and of composite samples. XRD pattern of pure PMMA is recognizable in graphs for the samples no. 1 and no. 2 by their broad amorphous maximums observed around $2 \theta=15^{\circ}, 30.2^{\circ}$ and $42.2^{\circ}$ as reported in the literature [14]. The specific broad diffraction peaks of pure PS around $2 \theta=20^{\circ}$ and $43^{\circ}$ observed in XRD pattern of sample no.3 are characteristic for pure PS [15]. From the graphs presented in Fig. 6., the BGO crystal characteristics are very good recognized both for samples no1 and no.3, but some peaks $\left(2 \theta=49.3^{\circ}, 79^{\circ}, 80.3^{\circ}\right.$ and $81.6^{\circ}$ are clearly visible only at XRD pattern of the sample no.3. The reasons are most probably that the particles of BGO at the top of the samples no.3 and no.1 are larger than those in sample no.2. and the broad XRD pattern peaks of PS do not coincide with those from pure crystal BGO.

\subsection{Raman spectra}

The Raman spectra of BGO single crystal and composite samples are presented in Fig. 7. In order to differentiate composite samples from polymers, observed modes were also compared with Raman spectra of pure PMMA and PS. Intensity modes at 553, 600, 730, 810, 965-999 (broad peak), 1180, 1237, $1450 \mathrm{~cm}^{-1}$ in the Raman spectra of samples no.1 and no.2 are characteristic for pure PMMA as it is presented in literature [16,17]. In Raman spectra of sample no.3 intensity modes at 366, 405, 621, 796, 1001, 1031, 1450 and $1584 \mathrm{~cm}^{-1}$, belong to pure PS, as it is described in Refs. [18-20].

The frequencies of the peaks observed in Raman spectra of the yellow $\mathrm{Bi}_{12} \mathrm{GeO}_{20}$ single crystals and the symmetry types of

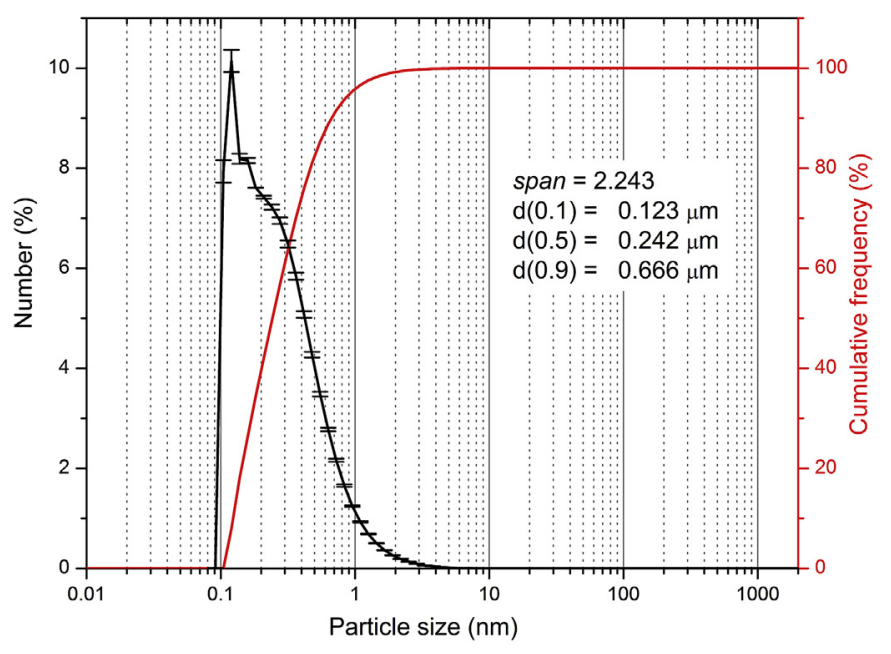

Fig. 1. Particle size distribution of BGO powder. corresponding vibrations are presented in the first and second column of the Table 2 based on the results presented in previous article [13] and literature [21]. The registered intensity modes for composite samples are presented in Table 2, where notation $s$ indicate that the peak is of low intensity or hardly to differentiate from the broad peak of the pure polymer.

In Raman spectra of the sample no. 1 the intensity modes are weakly perceived. Sample no. 2 have two well defined intensity modes and they are 269 and $538 \mathrm{~cm}^{-1}$ both of symmetry A which shows "breathing" of $\mathrm{Bi}$ and $\mathrm{O} 1$ and $\mathrm{O} 2$ atoms [13]. In sample no.2 the other intensity modes of symmetry A as well as of other symmetry types are observed. Sample no.3 have best observable intensity maximum at $620 \mathrm{~cm}^{-1}$, of symmetry E, which shows Bi and $01,02,03$ vibrations elongating the cluster along either $<100>$ or $<001>,<100>$ or $<010>$, respectively.

\subsection{Optical transmission spectra}

The described composite samples were prepared with high mass fraction in order to obtain XRD and Raman measurements that clearly distinguish BGO particles from polymer matrix and in this way investigate whether powder particles in such composites keep their crystal characteristics.

Optical transmission spectra were measured for the single crystal BGO plate, pure polymer samples and composite samples.

Single crystal plates of size $4 \mathrm{~mm} \times 4 \mathrm{~mm} \times 10 \mathrm{~mm}$ were cut from the boule with cutting plane perpendicular to the crystal growth direction and mechanically and chemically polished. The transmission spectrum of such a plate is presented in Fig. 8a). The pure BGO crystal plate is almost not transparent for wavelengths less than $500 \mathrm{~nm}$. This is in agreement with literature [22], since the energy gap of crystal BGO is about $3.2 \mathrm{eV}$ and the yellow color of this crystal is due to a broad absorption shoulder in the photon energy range from $2.3 \mathrm{eV}$ to $3.2 \mathrm{eV}$ (corresponding to the wavelengths from 539 to $387 \mathrm{~nm}$ ). At the same graph in Fig. 8a) the optical spectra of pure PMMA and pure PS polymer $0.6 \mathrm{~mm}$ thick plates are presented, too.

Optical transmission spectra of composite samples are presented in Fig. 8b). All presented spectra are normalized to the air transmission spectra.

The similar shapes of spectra for the samples no.1 and no. 2 reassemble to pure PMMA measured spectra. Although the samples no. 1 and no. 2 have the same initial BGO mass fraction $1 \mathrm{wt} \%$ the real mass fraction of the sample no.1 is most probable smaller than initial because of the filtering during preparation. Particles in sample no.2 have smaller sizes then those in sample no.1, according to the presented SEM analysis (paragraph 3.2.), so the light scattering is more intensive in this sample and since its real mass fraction is higher, its transmission is worse. Sample no.3 has low but almost linear transmission spectra versus wavelength.

The composite samples, thanks to polymer matrix, have wider transmission spectra than the BGO crystal. This allows using this material for double-wavelength techniques, where one of the wavelengths is chosen to be outside BGO transmission spectra. In case of using this material for fiber optic sensors this signal can be used as the normalization signal which compensates the effects of fiber bending and vibration.

\subsection{The calculations of composite sample transmission}

The described composite samples were prepared with high mass fraction, as previously explained and for this reason their measured transmissions were low, as expected. Those measured values were used therefore for comparison with calculated, based 


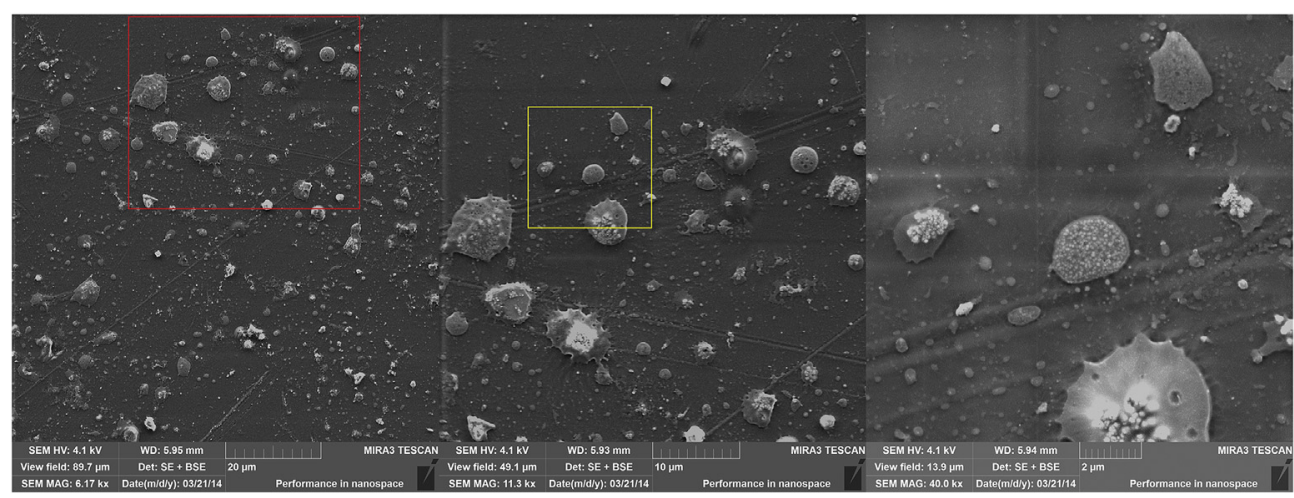

Fig. 2. SEM micrographs of the sample no.1 with different magnifications.

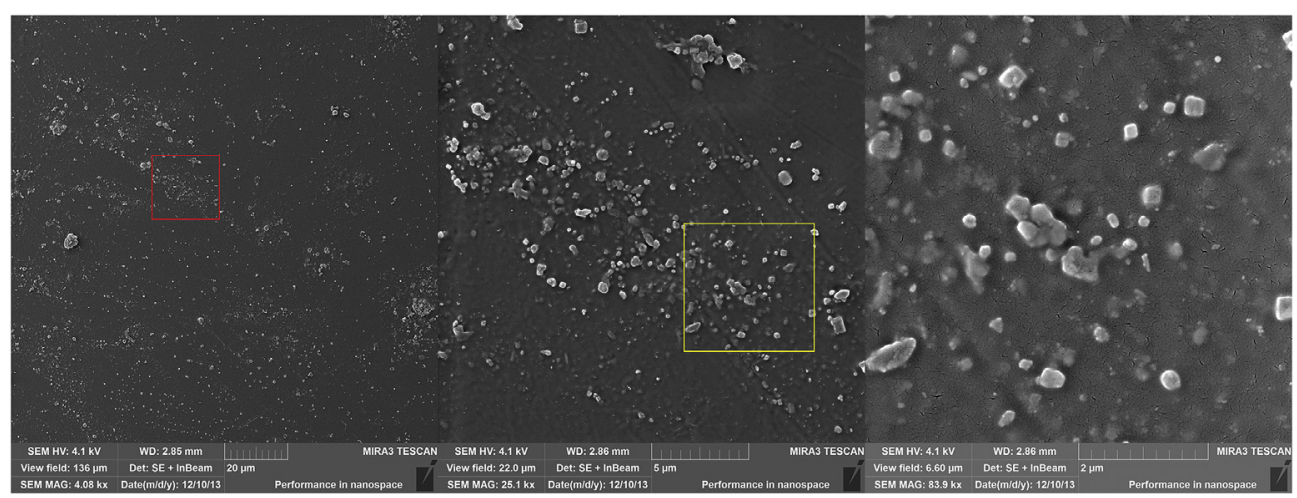

Fig. 3. SEM micrographs of the sample no.2 with different magnifications.

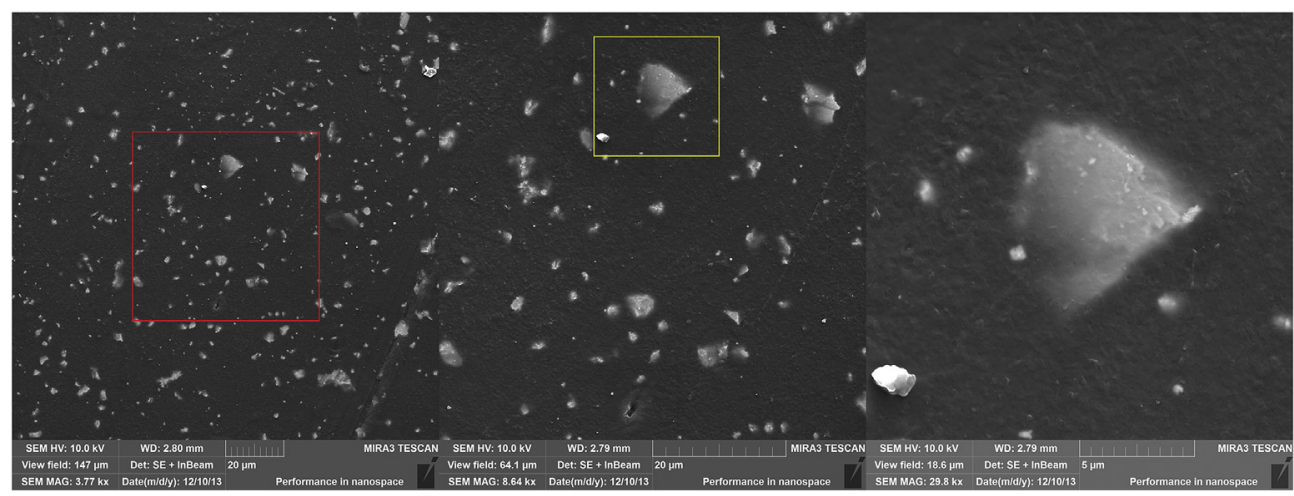

Fig. 4. SEM micrographs of the sample no.3 with different magnifications.

on Mie scattering theory, in order to check at what extent they correlate and if it is possible to predict the initial mass fraction of BGO for preparing the samples with sufficient transmission for the optical applications.

The electromagnetic radiation incident at the particle can be partially scattered and partially absorbed and the transmission of a material with dispersed particles depends on both processes. The amount of scattered and absorbed energy related to the incident is expressed in terms on scattering and absorption cross sections. The total energy loss based of both processes is called extinction and it is presented by extinction cross section as a sum of scattering and absorption cross sections. In the case of non-absorbing medium the scattering and extinction cross section are the same. One way of presenting both propagating and absorbing properties of a material for some kind of electromagnetic radiation is to introduce complex index of refraction. The existence of imaginary part shows that the material is absorbing for that kind of electromagnetic radiation and its value directly influences the absorption coefficient of the material.

The scattering cross section is ratio of scattered radiation power on the particle and incident intensity of radiation. Mie scattering theory presents the solution for the electromagnetic scattering by a sphere of radius $R$ embedded in a homogeneous and isotropic medium illuminated by a plane wave. If the index of refraction of a particle material is $n_{\mathrm{p}}$, and of medium $n_{\mathrm{m}}$, for some electromagnetic radiation of wavelength $\lambda_{0}$ in vacuum the scattering cross section depends on: size parameter which compare dimension of a particle and medium wavelength, given as $\chi=2 \cdot \pi \cdot R \cdot n_{m} / \lambda_{0}$, for spherical 

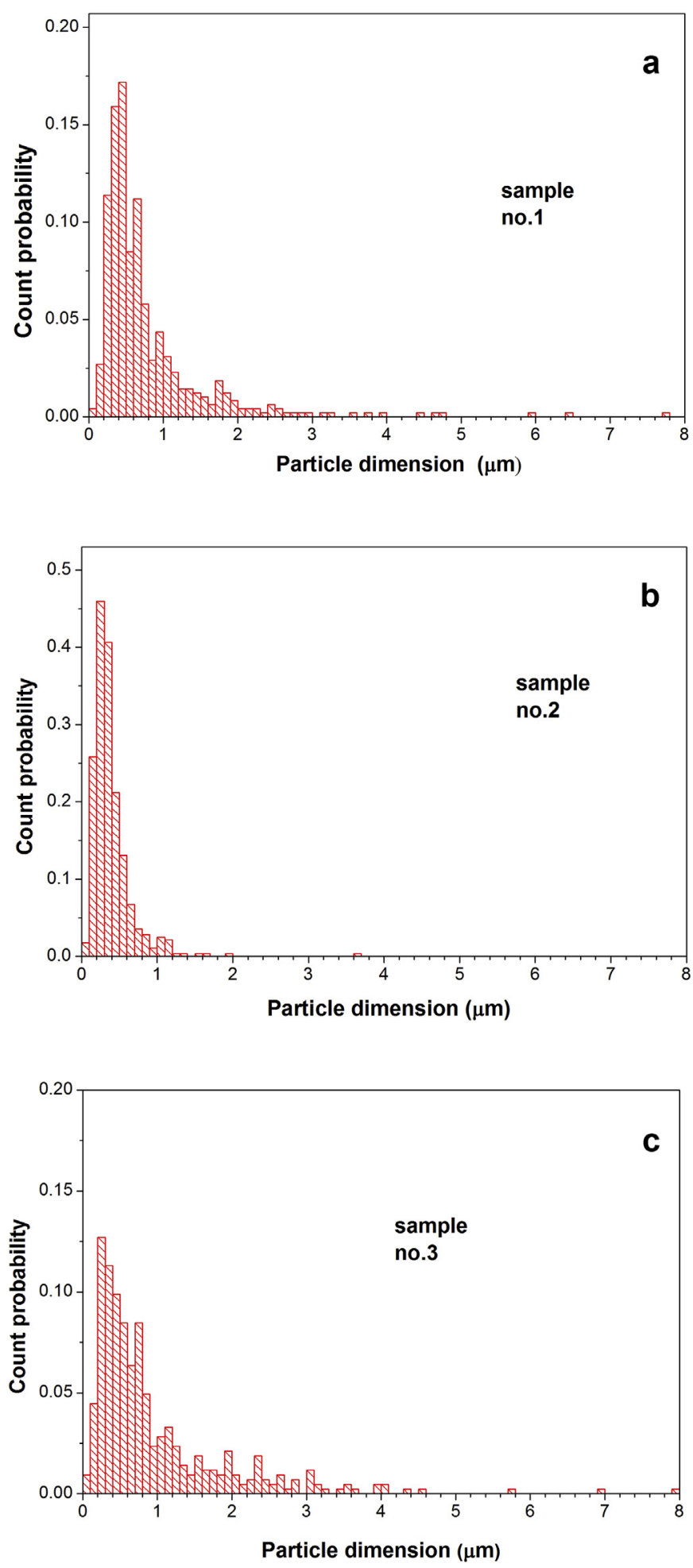

Fig. 5. Particle size distribution based on SEM analysis a) sample no.1, b) sample no.2 and c) sample no.3.

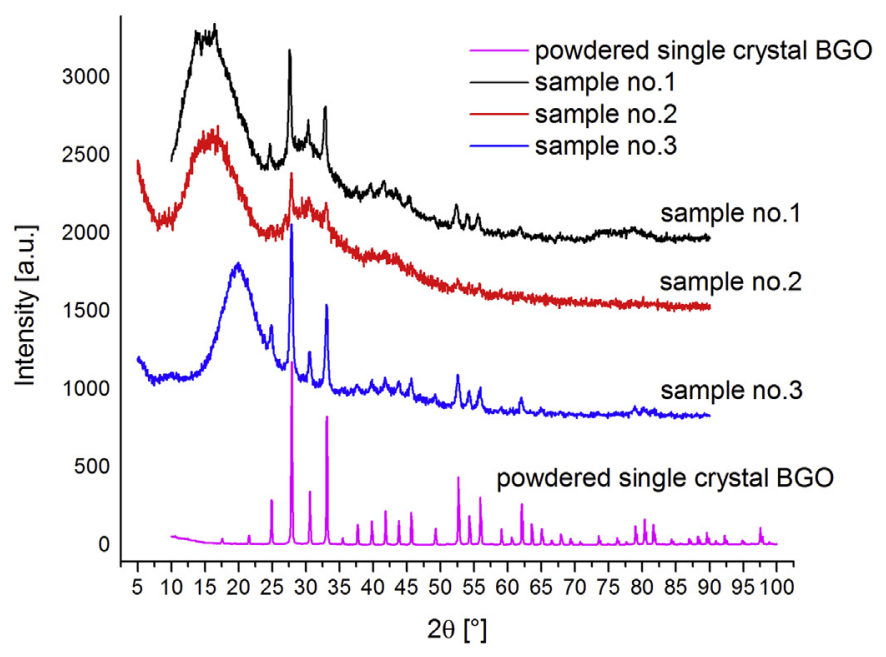

Fig. 6. XRD patterns of powdered BGO single crystal and composite samples.

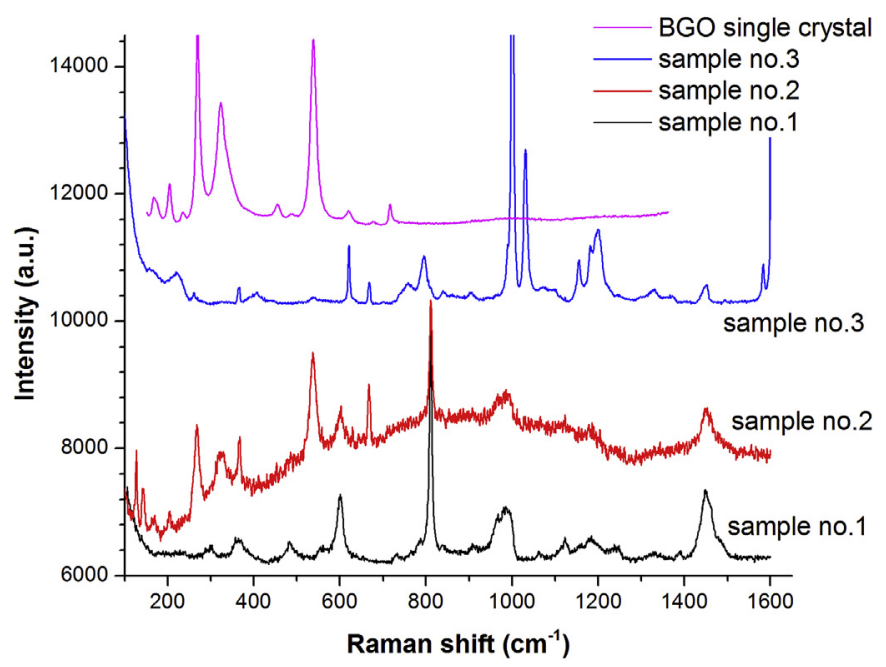

Fig. 7. Raman spectra of BGO single crystal and composite samples.

particle, and from the ratio of indices of refraction of particle and medium given as $\mathrm{m}=\mathrm{n}_{\mathrm{p}} / \mathrm{n}_{\mathrm{m}}$.

The calculations were done using Mie calculator software $[12,23]$ for spherical particles. Input data for the calculations were: mass density of PMMA $\rho_{\text {PMMA }}=1.18 \mathrm{~g} / \mathrm{cm}^{3}$, mass density of PS $\rho_{\mathrm{PS}}=1.0 \mathrm{~g} / \mathrm{cm}^{3}$ and mass density of BGO $\rho_{\mathrm{BGO}}=9.23 \mathrm{~g} / \mathrm{cm}^{3}$. Based on those data the total volume fraction $f_{\mathrm{V}}$ of BGO in composite samples is calculated based on equation (1):

$f_{V}=\frac{\rho_{\text {pol }} \cdot f_{W}}{\left(1-f_{W}\right) \cdot \rho_{B G O}+\rho_{p o l} \cdot f_{W}}$

In the equation (1) $\rho_{\text {pol }}$ denotes mass density of used polymer ( $\rho_{\text {PMMA }}$ or $\rho_{\mathrm{PS}}$ ) and $f_{\mathrm{W}}$ denotes mass fraction of BGO powder in the

Table 1

Statistics parameters of PSD in composite samples based on SEM analysis.

\begin{tabular}{|c|c|c|c|c|c|c|c|}
\hline Sample & $N$ & $\min d, \mu \mathrm{m}$ & $\max d, \mu \mathrm{m}$ & mean $d, \mu \mathrm{m}$ & st. dev., $\mu \mathrm{m}$ & $\mathrm{d}(0.5), \mu \mathrm{m}$ & $\mathrm{d}(0.9), \mu \mathrm{m}$ \\
\hline no.1 & 484 & 0.044 & 9.34 & 0.802 & 0.897 & 0.532 & 1.65 \\
\hline no.2 & 483 & 0.082 & 3.61 & 0.389 & 0.280 & 0.325 & 0.659 \\
\hline no.3 & 425 & 0.071 & 7.93 & 0.960 & 0.796 & 0.639 & 2.25 \\
\hline
\end{tabular}


Table 2

Raman frequencies observed in single crystal BGO [13] and samples.

\begin{tabular}{|c|c|c|c|c|}
\hline BG0 single crystal wave number, $\mathrm{cm}^{-1}$ & Symmetry type & Sample no. 1 wave number, $\mathrm{cm}^{-1}$ & Sample no.2 wave number, $\mathrm{cm}^{-1}$ & Sample no. 3 wave number, $\mathrm{cm}^{-1}$ \\
\hline 168 & A & & $166-170$ & \\
\hline 190 & $\mathrm{~F}(\mathrm{LO})$ & $190 s$ & & \\
\hline 204 & $\mathrm{~F}(\mathrm{TO})$ & & 204 & \\
\hline 234 & E & $234 \mathrm{~s}$ & & \\
\hline 268 & A & & 269 & $269 s$ \\
\hline 322 & A & $322 s$ & 322 wide peak & \\
\hline 454 & $\mathrm{E}$ & & $454 \mathrm{~s}$ & \\
\hline 486 & $\mathrm{~F}(\mathrm{TO}+\mathrm{LO})$ & $488 \mathrm{~s}$ & $488 \mathrm{~s}$ & \\
\hline 538 & A & & 538 & 538 \\
\hline 619 & E & $620 \mathrm{~s}$ & & 620 \\
\hline 677 & $\mathrm{~F}(\mathrm{TO})$ & $676 s$ & $677 s, 682 \mathrm{~s}$ & \\
\hline 715 & A & & & $716 s$ \\
\hline
\end{tabular}

s-small, hardly visible intensity peak.

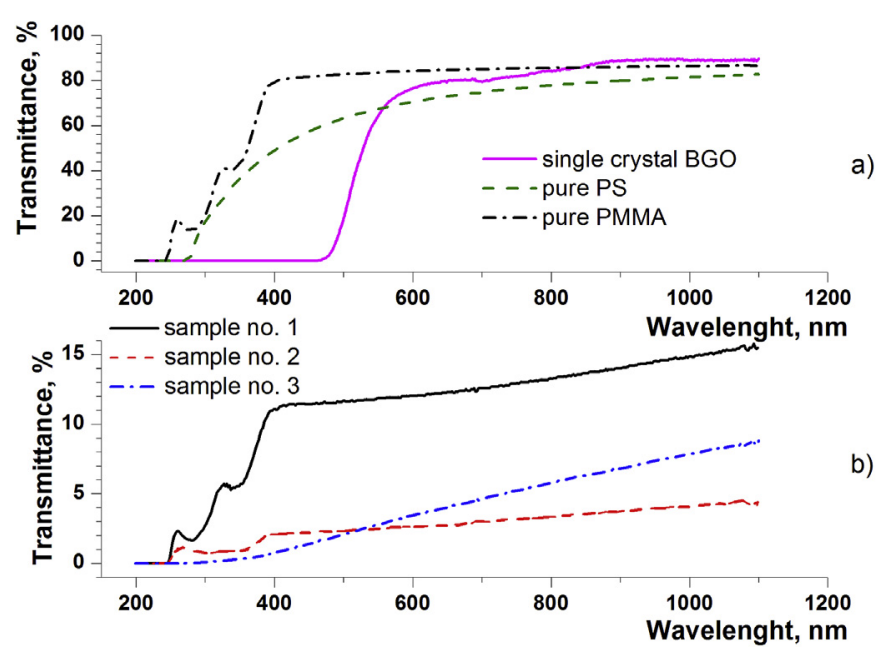

Fig. 8. Transmission spectra of: a) single crystal BGO, pure PMMA and pure PS, b) composite samples.

samples that was 0.01 (i.e. $1.0 \mathrm{wt} \%$ ). Spectral dependences of index of refraction of the polymers and BGO were taken from the refractive index database [24]. Since the data for BGO are only available in the spectral range from 450 to $700 \mathrm{~nm}$ and the crystal is not or low transparent for wavelengths below $500 \mathrm{~nm}$, the calculations of transmission of samples are derived for $500 \mathrm{~nm}, 600 \mathrm{~nm}$ and $700 \mathrm{~nm}$.

The scattering cross sections $\sigma_{\mathrm{iM}}$ were calculated for spherical particles of diameter $d_{\mathrm{i}}$, where $d_{\mathrm{i}}$ was taken in the range $100 \mathrm{~nm}$ $0.8 \mu \mathrm{m}$ at $20 \mathrm{~nm}$ steps and in the range $0.8 \mu \mathrm{m}-10 \mu \mathrm{m}$ at $0.1 \mu \mathrm{m}$ steps for each of those three wavelengths.

Assumed that the number of particles with diameter $d_{\mathrm{i}}$ is $N_{\mathrm{i}}$, the total volume of those particles was calculated as $N_{i} V_{i}=N_{i} \cdot \pi \cdot \frac{d_{i}^{3}}{6}$. Using total volume fraction of BGO in the sample from equation (1) the number concentration $n_{\mathrm{i}}$ of particles having diameter $d_{\mathrm{i}}$ is:

$n_{i}=\frac{f_{V} \cdot N_{i}}{\sum_{i=1}^{K}\left(N_{i} \cdot V_{i}\right)}$

where $K$ is the number of different diameters of particles used in calculations. The scattering coefficient of the polymer composite sample at one wavelength $\gamma_{S}$ is than obtained as:

$\gamma_{s}=\sum_{i=1}^{K} n_{i} \sigma_{i M}$
Since the imaginary parts of indices of refraction for BGO and polymers were zero, obtained scattering coefficients are the same as the extinction coefficients of the samples.

If two parallel planes, at distance $L$, inside a polymer composite are imagined and if $I_{0}$ is the intensity of light incoming at first plane and $I$ is intensity of transmitted light reaching the second plane, the transmission coefficient $\mathrm{T}$ can be calculated as:

$T=\frac{I}{I_{0}}=e^{-\gamma_{S} \cdot L}$

Thus obtained value $\mathrm{T}$ in equation (4) is the transmission or transmission coefficient of the composite sample normalized to the transmission of the pure polymer sample of the same width and for the same wavelength. In order to calculate the transmission spectra of a sample normalized to the air, calculated transmission $\mathrm{T}$ is multiplied with the measured transmission coefficient of corresponding pure polymer sample for that wavelength. In case of comparing composite samples based on the same polymer matrix it is more often to measure or calculate transmission normalized to transmission of pure polymer. Since our composite samples are based on different polymer matrices, their transmissions, normalized to the air, are compared.

The calculations based on the described procedure were applied to various PSD:

a) In order to evaluate the transmission coefficients in an "ideal" case, i.e. the case that particle size distribution in the polymer composite is the same as in the powder, the values for $N_{\mathrm{i}}$ and $d_{\mathrm{i}}$, were taken from the paragraph 3.1. The transmissions were calculated for $L=0.6 \mathrm{~mm}$ thick plates and the results were presented also in Table 3 All calculated values were higher than measured since the particles were not ideally spherical and ideally dispersed. For the used mass fraction of $1 \mathrm{wt} \%$, the maximum obtained values are around $17 \%$, which means that input mass fraction of such BGO particles should be lower in order to get better transmissions. The measured transmission values for sample no.2 and no.3 (from Table 3) are five to six times lower from those maximums. Thus, to achieve sample the transmission of $50 \%$ of those samples, the number concentration of particles should be about five times lower (based on equations (3) and (4)) for both samples, which corresponds to particle mass fraction of $0.2 \mathrm{wt} \%$.

b) The calculations based on PSD from SEM analysis of polymer samples (results presented in paragraph 3.2.) were calculated, too. In these calculations the measured longest dimension of a particle was assumed as a diameter $d_{\mathrm{i}}$ of the particle. The results are presented in Table 3. The obtained values for samples no. 1 and no.3 differ significantly from measured values and those for sample no.2 show the best match, slightly higher than measured.

One of the reasons for this mismatch of measured and calculated 
values is that PSD based on SEM analysis, which is in fact twodimensional and relevant to the top of sample, was used as volume PSD. The PSD inside the sample could be different from that on the top. It seems from comparison of calculated and measured values that real partition of smaller particles (size up to $0.5 \mu \mathrm{m}$ diameter) is higher than from SEM analysis since their presence increases scattering volume and hence decreases transmission. The other reason for this mismatch is that in this type of modelling the aggregates are taken as spheres of pure crystal BGO, not as a group of very close connected particles. The light scattering process is more intensive on those particles group than on the homogeny sphere of the same diameter. For these reason, the aggregates have more significant influence on scattering loss, and some kind of their effective scattering cross sections are larger than obtained in this modelling.

The best match between calculated and measured results is for sample no. 2 since the most of the powder volume in it is uniformly dispersed in small particles.

Sample no. 1 has better measured transmission than sample no.2 because its real particle mass fraction is in fact less than $1 \mathrm{wt} \%$ due to filtering On the other hand its larger particles and aggregates whose influence increases the transmission are not only round but sometimes with very complex structure. In calculations based on maximum particle length taken as a diameter of sphere, calculated volumes of large particles could be much higher than real and thus the calculated small particle size mass concentration based on (2) is lower than real. Since the input mass fraction of sample no. 1 is changed due to filtering during preparation procedure, the real mass fraction for the sample no.1 is not known, as well as, all the input values based on it. So with not known number concentrations of particles, as a main input data, the calculated influence of particle shape could not give valuable information for comparison with measuring data.

The dispersed particles in sample no. 3 were mostly not spherical, so the concentration of smaller particles and their scattering cross sections were calculated with the errors. The scattering cross section calculations for non-spherical arbitrary shape particles are much more complicated than those based on Mie theory for spherical ones. There are lot of researches that compare the scattering cross sections of non-spherical and some kind of equivalent spherical particle [25-28]. They have presented their results through comparing graphs or by correction factors that show how to choose equivalent spherical particle that have similar scattering cross section as corresponding non-spherical one.

The dispersed particles and aggregates in sample no.3 could be in the first approximation taken as spheroids. The longest axis of such spheroids $c_{\mathrm{i}}$ equals the half of their measured longest dimension $d_{\mathrm{i}}$ and their perpendicular axis $a_{i}$ is in average twice shorter than $c_{\mathrm{i}}$ i.e. $d_{\mathrm{i}}=2 c_{\mathrm{i}}=4 a_{\mathrm{i}}$. In this case the spheroid particle volume can be calculated as $V_{i S R D}=\frac{\pi d_{i}^{2}}{24}$. In literature [28] the scattering efficiency $Q_{S R D}$ for spheroid and $Q_{S}$ for related sphere, i.e. sphere whose radius equals the length the smaller axis of the spheroid were presented for different size parameters, and for different elongation factors $\gamma=c_{\mathrm{i}} / a_{\mathrm{i}}$ of the spheroid. For the particles in sample no. 3 this factor was taken as $\gamma=2$. The size parameters for wavelengths $\lambda_{0}=500,600$ and $700 \mathrm{~nm}$ were calculated as $\chi_{i}=\frac{2 \pi \cdot n_{P S}\left(\lambda_{0}\right)}{\lambda_{0}} a_{i}$, where $n_{P S}\left(\lambda_{0}\right)$ was index of refraction of PS for the wavelength $\lambda_{0}$. From the PSD based on SEM analysis for the sample no. 3 values of $\chi_{i}$ were mostly bigger than 3 except for the particles that have $d_{\mathrm{i}}$ smaller than $0.4 \mu \mathrm{m}$. The relation of scattering efficiencies for the spheroid and the sphere versus $\chi_{i}$ taken from the literature (graph in Fig. 12b from Ref. [28]) was fitted with the curve

$Q_{\text {rel }}=\frac{Q_{S R D}}{Q_{S}}=A \cdot \chi_{i}^{-B}$

for $0.1 \leq \chi_{\mathrm{i}} \leq 4$, where $\mathrm{A}=1.59903$, and $\mathrm{B}=0.32823$. For $\chi_{\mathrm{i}}>4$, based on the same literature [28], $Q_{r e l}=1$.

Since the cross section of the spheroid is two times bigger than the cross section of the sphere in the direction of the incident light and based on the definition of scattering efficiency [28] scattering cross section for the spheroid particle $\sigma_{\mathrm{iSRD}}$ was calculated as

$\sigma_{i S R D}=2 \cdot Q_{r e l} \cdot \sigma_{i M}$

The equations from (2)-(4) were applied for spheroids in a way that $V_{\mathrm{iSRD}}$ is used instead of $V_{\mathrm{i}}$ and $\sigma_{\mathrm{iSRD}}$ instead of $\sigma_{\mathrm{iM}}$ for every wavelength. The transmissions of sample no.3 based on spheroid particles are presented in Table 3 too, and are much smaller than obtained for spherical particles, but still higher than measured, since real particle shape is more complex than spheroidal and the influence of aggregates is not taken into account, probably because the SEM analysis in the case of sample no. 3 did not represent real PSD in the whole sample.

c) Another type of idealized backward calculation based on Mie scattering for spherical particle was done. It was supposed that the crystal BGO powder was ideally milled into spherical particles of the same diameter $d_{\mathrm{eff}}$, and so the concentration of particles with the volume fraction $f_{\mathrm{V}}$ became $n_{\text {eff }}=\frac{f_{\mathrm{V}}}{\pi \frac{d^{3}}{f_{f}}}$. Scattering coefficient of such material is $\gamma_{\text {Seff }}=n_{\text {eff }} \bullet \sigma_{\text {ieff }}$ and using previously calculated scattering cross sections $\sigma_{\text {iM }}$ for different diameters as $\sigma_{\text {ieff, the }}$ dependence $\gamma_{S \text { eff }}$ versus $d_{\text {eff }}$ could be calculated based on (3) for each wavelength. Comparing those values with obtained $\gamma_{S}$ from measured transmission using equation (4), $d_{\mathrm{eff}}$ for each sample

Table 3

Measured and calculated optical transmission of the composite samples for three wavelengths.

\begin{tabular}{|c|c|c|c|c|c|}
\hline \multirow[t]{3}{*}{ Sample } & \multirow[t]{3}{*}{ Method of obtaining results } & & \multicolumn{3}{|c|}{ Transmission T,\% } \\
\hline & & & \multicolumn{3}{|c|}{ Wavelength $\lambda_{0}, \mathrm{~nm}$} \\
\hline & & & 500 & 600 & 700 \\
\hline \multirow[t]{3}{*}{ no.1 } & Measured & & 11.63 & 12.03 & 12.56 \\
\hline & Calculated (Mie-spheres) & Based on powder PSD & 16.91 & 16.86 & 17.42 \\
\hline & & Based on PSD from SEM & 42.6 & 42.5 & 42.1 \\
\hline \multirow[t]{3}{*}{ no.2 } & Measured & & 2.3 & 2.6 & 3.0 \\
\hline & Calculated (Mie-spheres) & Based on powder PSD & 16.9 & 16.9 & 17.4 \\
\hline & & Based on PSD from SEM & 4.46 & 3.9 & 3.96 \\
\hline \multirow[t]{4}{*}{ no.3 } & Measured & & 3.3 & 4.6 & 6.2 \\
\hline & Calculated (Mie-spheres) & Based on powder PSD & 16.8 & 18.4 & 19.1 \\
\hline & & Based on PSD from SEM & 31.9 & 34.7 & 37.3 \\
\hline & & Based on PSD from SEM and spheroid correction & 14.6 & 14.8 & 16.1 \\
\hline
\end{tabular}


could be estimated for those three wavelengths. Averaging such obtained values over all wavelengths the calculated $d_{\text {eff }}$ for sample no. 1 is $1.41 \pm 0.11 \mu \mathrm{m}$, for sample no. 2 the corresponding value is $0.87 \pm 0.11 \mu \mathrm{m}$ and for sample no. 3 is $0.79 \pm 0.05 \mu \mathrm{m}$. The value of $d_{\text {eff }}$ for sample no.2 is similar to its $d(0.9)$ value from Table 1 . In case of sample no.3 obtained $d_{\text {eff }}$ is significantly smaller of its d (0.9) from SEM analysis (Table 1) and this could indicates that the volume fraction of small particles is higher than evaluated from SEM analysis or the scattering of aggregates is more similar to scattering at small particles.

\section{Conclusion}

In this work it is shown that solution casting method is suitable for preparing polymer composites with milled BGO powder as filler. In such composites the BGO crystal structure should be preserved, the particles uniformly distributed and the samples enough transparent for potential electro-optical and magneto-optical applications.

The investigations of three prepared samples based on two polymer matrix (PMMA and PS) and two solvents (acetone and chloroform) pointed out that the dispersion of particles was very good in both PMMA and PS samples with chloroform as solvent but particles and agglomerate sizes were smaller in PMMA. The dispersed particles and agglomerates in PMMA are also more round than in PS. Two methods of dispersion and deaggregation of BGO powder were used in this study (filtration and sonication) and next research will be focused on the application of ultrasonic irradiation.

Powdered BGO particles keep their crystal characteristics in the composites and XRD measurements best recognize BGO characteristics in PS sample (no.3) while Raman spectroscopy was more efficient in detecting vibrational modes in the PMMA sample with chloroform as solvent.

Optical transmission of prepared samples was low since the BGO particles mass fraction was intentionally high (1 wt\%) in order to have XRD and Raman measurements that clearly distinguish BGO particles in polymer matrix. The transmission of samples based on Mie theory scattering calculations for spherical particles was obtained based on PSD in the BGO powder, and on SEM analysis of the samples. In case of ideal powder particles dispersion when their PSD in the composite sample is the same as in the powder, it is shown that maximum obtained transmission would be $17 \%$, when particle mass fraction is $1 \mathrm{wt} \%$ and in order to increase the transmission of such prepared samples the mass fraction of BGO particles should be lower. The real transmission values for sample no.2 and no.3 are five to six times lower from those maximum. To achieve the transmission of $50 \%$, the number concentration of particles should be about five times lower for both samples, which corresponds to particle mass fraction of $0.2 \mathrm{wt} \%$.

When the calculations are based on SEM analysis the obtained values of PMMA/chloroform composite have good match with measured because the particle sizes are the smallest and mostly round. On contrary, in the PS polymer composite particles have irregular shapes, and the approximation with spheroids with elongation factor 2 gave better results but still higher than measured.

The difference between calculated and measured transmission values is caused mainly because this type of modelling treats aggregates as pure crystal BGO spheres or spheroids, not as groups of very close connected particles. The light scattering process is more intensive on those multi-particles aggregates than on the homogeny crystal particles of the same dimension. So, comparing of calculating and measured transmission could be used to indicate level of agglomeration of particles in the samples.

The same Mie scattering calculations were used for some sort of backward modelling in order to find the equivalent diameter of the identical BGO powdered spherical particles that would gave the same transmission as prepared samples, with the same BGO mass fraction.

\section{Acknowledgments}

This work was supported by the Ministry of Science and Technological Development of the Republic of Serbia, Projects No. TR 34011 and III 45003.

\section{Appendix A. Supplementary data}

Supplementary data related to this article can be found at http:// dx.doi.org/10.1016/j.jallcom.2016.10.140.

\section{References}

[1] V.M. Skorikov, Yu F. Kargin, A.V. Egorysheva, V.V. Volkov, M. Gospodinov Growth of sillenite-structure single crystals, Inorg. Mater. 41 (1) (2005) S24-S46.

[2] N.C. Deliolanis, I.M. Kourmoulis, G. Asimellis, A.G. Apostolidis, E.D. Vanidhis N.A. Vainos, Direct measurement of the dispersion of the electrogyration coefficient of photorefractive $\mathrm{Bi}_{12} \mathrm{GeO}_{20}$ crystals, J. Appl. Phys. 97 (2005) 023531.

[3] R.A. Ganeev, A.I. Ryasnyansky, B. Palpant, S. Debrus, Third-order nonlinearities of $\mathrm{Bi}_{12} \mathrm{GeO}_{20}$ crystal measured by nanosecond radiation, J. Appl. Phys. 97 (2005) 104303.

[4] E. Weidner, G. Pauliat, G. Roosen, Wavefront buffer memory based interferometric camera with a photorefractive crystal as the active medium, J. Opt. A Pure Appl. Opt. 5 (6) (2003) S529.

[5] S. Sayuri Sato, J.C. Santos, Modeling residual thermal stress-induced integrated optical waveguides on $\mathrm{Bi}_{12} \mathrm{GeO}_{20}$ substrate for electrooptic modulation application, in: SBMO/IEEE MTT-S International Microwave \& Optoelectronics Conference (IMOC 2009), 2009, pp. 669-672.

[6] O. Peña-Rodríguez, J. Olivares, I. Bányász, Optical properties of crystalline and ion-beam amorphsed $\mathrm{Bi}_{12} \mathrm{GeO}_{20}$ : relevance for waveguide applications, Opt. Mater. 47 (2015) 328-332.

[7] P.M. Mihailovic, S.J. Petricevic, J.B. Radunovic, Compensation for temperaturedependence of the faraday effect by optical activity temperature shift, IEEE Sens. J. 13 (2) (2013) 832-837, http://dx.doi.org/10.1109/JSEN.2012.2230322.

[8] L. Labrunie, G. Pauliat, G. Roosen, J. Launay, Simultaneous acquisition of pi/2 phase-stepped interferograms with a photorefractive $\mathrm{Bi}_{12} \mathrm{GeO}_{20}$ crystal: application to real-time double-pulse holography, Opt. Lett. 20 (15) (1995) 1652-1654.

[9] D.R. Paul, L.M. Robeson, Polymer nanotechnology: nanocomposites, Polymer 49 (2008) 3187-3204

[10] D. Stojanovic, A. Orlovic, S. Markovic, V. Radmilovic, Petar S. Uskokovic R. Aleksic, Nanosilica/PMMA composites obtained by the modification of silica nanoparticles in a supercritical carbon dioxide-ethanol mixture, J. Mater. Sci. 44 (2009) 6223-6232.

[11] R.M. Dukali, I. Radovic, D.B. Stojanovic, P.S. Uskokovic, N. Romcevic, V. Radojevic, R. Aleksic, Preparation, characterization and mechanical properties of $\mathrm{Bi}_{12} \mathrm{SiO}_{20}-$ PMMA composite films, J. Alloys Compd. 583 (2014) 376-381.

[12] D.B. Stojanović, L. Brajović, A. Orlović, D. Dramlić, V. Radmilović, P.S. Uskoković, R. Aleksić, Transparent PMMA/silica nanocomposites containing silica nanoparticles coating under supercritical conditions, Prog. Org. Coat. 76 (4) (2013) 626-631.

[13] Z.Ž. Lazarević, P. Mihailović, S. Kostić, M.J. Romčević, M. Mitrić, S. Petričević J. Radunović, N.Ž. Romčević, Determination of magneto-optical quality and refractive index of bismuth germanium oxide single crystals grown by Czochralski, Opt. Mater. 34 (11) (2012) 1849-1859.

[14] R. Baskaran, S. Selvasekarapandian, N. Kuwata, J. Kawamura, T. Hattori, Conductivity and thermal studies of blend polymer electrolytes based on PVAc-PMMA, Solid State Ionics 177 (2006) 2679-2682.

[15] N. Zhang, X. Yu, J. Hu, F. Xue, E. Ding, Synthesis of silver nanoparticle-coated poly(styrene-co-sulfonic acid) hybrid materials and their application in surface-enhanced Raman scattering (SERS) tags, RSC Adv. 3 (2013) $13740-13747$.

[16] B. Schneider, J. Štokr, P. Schmidt, M. Mihailov, S. Dirlikov, N. Peeva, Stretching and deformation vibrations of $\mathrm{CH}_{2}, \mathrm{C}\left(\mathrm{CH}_{3}\right)$ and $\mathrm{O}\left(\mathrm{CH}_{3}\right)$ groups of poly(methylmethacrylate), Polymer 20 (1979) 705-712.

[17] X. Xingsheng, M. Hai, Z. Qijing, Z. Yunsheng, Properties of Raman spectra and laser-induced birefringence in polymethyl methacrylate optical fibres, J. Opt. A Pure Appl. Opt. 4 (2002) 237-242.

[18] A. Palm, Raman spectrum of polystyrene, J. Phys. Chem. 55 (8) (1951) $1320-1324$.

[19] B. Jasse, L. Monnerie, Far-infrared and Raman spectra of polystyrene model molecules, J. Phys. D. Appl. Phys. 8 (1975), 863-861. 
[20] A. Serafim, R. Mallet, F. Pascaretti-Grizon, I-C. Stancu, D. Chappard, Osteoblastlike cell behavior on porous scaffolds based on poly(styrene) fibers, Biomed. Res. Int. 2014 (2014) 609319.

[21] S. Venugopalan, A.K. Ramdas, Raman spectrum of bismuth germanium oxide* Phys. Lett. 34A (1971) 9-10.

[22] R. Oberschmid, Absorption centers of $\mathrm{Bi}_{12} \mathrm{GeO}_{20}$ and $\mathrm{Bi}_{12} \mathrm{SiO}_{20}$ crystals, Phys. Stat. Sol.(a) 89 (1) (1985) 263-270.

[23] Mie scaterring by Scott Prahl, http://omlc.org/calc/mie_calc.html, 2016 (accessed 14.03.2016).

[24] Refractive index database, www.refractivelndex.info, 2015 (accessed 17.12.2015).
[25] M.I. Mishchenko, Electromagnetic scattering by nonspherical particles: a tutorial review, J. Quant. Spectrosc. Radiat. Transf. 110 (2009) 808-832.

[26] X. Li, Z. Chen, A. Taflove, V. Backman, Equiphase - sphere approximation for analysis of light scattering by arbitrarly shaped nonspherical particles, Appl. Opt. 43 (2004) 4497-4505.

[27] K. Muininen, T. Pieniluoma, Light Scattering by Gaussian random ellipsoid particles: first results with discrete dipole approximation, J. Quant. Spectrosc. Radiat. Transf. 112 (2011) 1747-1752.

[28] V. Rysakov, M. Ston, Light scattering by spheroids, J. Quant. Spectrosc. Radiat. Transf. 69 (2001) 651-665. 